\title{
Silodosin is effective for treatment of LUTS in men with BPH: a systematic review
}

\begin{abstract}
Hui Ding ${ }^{1}$, Wan $\mathrm{Du}^{2}$, Zi-Zhen Hou ${ }^{1}$, Han-Zhang Wang ${ }^{3}$ and Zhi-Ping Wang ${ }^{1}$
The aim of this study was to systematically review the evidence on the efficacy and safety of silodosin treatments on lower urinary tract symptoms (LUTS) in men with benign prostatic hyperplasia (BPH) from randomized controlled trials. We searched PubMed (1966December 2011), Embase (1974-December 2011) and the Cochrane Library Database (2011, Issue 12). The assessed outcome measures were the change from baseline for the International Prostate Symptom Score (IPSS), quality of life (QoL) score, peak urine maximum flow rate $\left(Q_{\max }\right), Q \circ L$ related to urinary symptoms and adverse effects. Two authors independently assessed the study quality and extracted data. All data were analysed using RevMan 5.1. The meta-analysis included four randomized controlled trials with a total of 2504 patients. The study durations were each 12 weeks. At the follow-up end points, the pooled results showed that the change from baseline for the silodosin group was significantly higher than the placebo group for the IPSS, QoL score and $\mathbf{Q}_{\max }$ (mean difference $(M D)=-2.78, P<0.00001 ; M D=-0.42, P=0.004 ; M D=1.17, P<0.00001$, respectively) and patients felt more satisfied with QoL related to urinary symptoms in the silodosin group than the placebo group. Ejaculation disorder was the most commonly reported adverse effect. The pooled results also showed that the silodosin group was superior to the $0.2 \mathrm{mg}$ tamsulosin group with respect to the IPSS and QoL score (IPSS: MD =-1.14, $P=0.02$; QoL score: $M D=-0.26, P=0.02$ ) and inferior to the $0.2 \mathrm{mg}$ tamsulosin group with respect to $Q_{\max }(M D=-0.85, P=0.01)$. In contrast, there was no significant difference in the incidence of ejaculation disorder and dizziness between the silodosin and $0.2 \mathrm{mg}$ tamsulosin groups. The current meta-analysis suggested that silodosin is an effective therapy for LUTS in men with BPH and is not inferior to $0.2 \mathrm{mg}$ tamsulosin.
\end{abstract}

Asian Journal of Andrology (2013) 15, 121-128; doi:10.1038/aja.2012.102; published online 10 December 2012

Keywords: benign prostatic hyperplasia (BPH); KMD-3213; lower urinary tract symptoms (LUTS); silodosin; tamsulosin; systematic review; meta-analysis

\section{INTRODUCTION}

Histologically, benign prostatic hyperplasia (BPH) is a non-malignant enlargement of the prostate caused by cellular hyperplasia of both glandular and stromal elements. ${ }^{1}$ However, it is clinically characterised by lower urinary tract symptoms (LUTS) (urinary frequency, urgency, a weak and intermittent stream, the need to strain, a sense of incomplete emptying and nocturia), and can lead to complications, including acute urinary retention. ${ }^{2}$ The development of human BPH correlates with increasing age, ${ }^{3}$ and LUTS are fairly common after the age of 50 years. ${ }^{4}$ As is well known, the treatment options include alpha-blockers, five alpha-reductase inhibitors, transurethral resection of the prostate, transurethral microwave thermotherapy and herbal treatments (saw palmetto and $\beta$-sitosterol plant extracts). According to the EAU 2011 guidelines, alpha-blockers are currently the preferred first-line therapy for all men with moderate or severe LUTS/BPH. ${ }^{5}$ Alpha-blockers for BPH include alfuzosin, prazosin, doxazosin, tamsulosin and terazosin, which are selective alpha ${ }_{1}$-adrenergic blockers. ${ }^{6-10}$

Compared with non-selective alpha ${ }_{1 \mathrm{~A}}$-adrenoceptor (AR) blockers, drugs with a high selectivity for alpha ${ }_{1 \mathrm{~A}}-\mathrm{AR}$ may be more prostate-specific and maintain a therapeutic response in the treatment of symptomatic BPH with less effect on blood pressure and fewer cardiovascular side effects. ${ }^{11,12}$ Therefore, to reduce the risk of cardiovascular side effects in patients with $\mathrm{BPH}$, an ideal alpha-blocker should increase the alpha ${ }_{1 \mathrm{~A}}$-receptor subtype selectivity and reduce the non-alpha ${ }_{1 \mathrm{~A}}$-receptor subtype selectivity. In the past few years, silodosin (KMD-3213), a new highly selective alpha $_{1 \mathrm{~A}}$-AR antagonist, was developed by Kissei Pharmaceutical Co., Ltd (Matsumoto, Japan). Silodosin was demonstrated to have a higher selectivity for the alpha ${ }_{1 \mathrm{~A}}-\mathrm{AR}$ subtype than tamsulosin hydrochloride, naftopidil or prazosin hydrochloride, and the alpha $_{1 A^{-}}$-to-alpha $a_{1 \mathrm{~B}}$ binding ratio of silodosin is $162: 1 .^{13}$ These results suggested that silodosin may have more beneficial effects on the symptoms associated with $\mathrm{BPH}$ and minimal effects on blood pressure. Several randomized controlled trials have reported the clinical effectiveness and safety of silodosin for BPH. ${ }^{14-16}$ To date, however, there have been no systematic reviews or metaanalyses that including randomized controlled trials (RCTs) to determine the effectiveness and safety of silodosin for BPH. Therefore, the aim of this meta-analysis is to evaluate the efficacy and safety of silodosin for LUTS in men with BPH for providing more reliable evidence for the use of silodosin. 


\section{MATERIALS AND METHODS}

\section{Search strategy}

Literature searches were performed to identify RCTs of silodosin for the treatment of LUTS in men with BPH. The following databases were used: PubMed (1966-December 2011), Embase (1974-December 2011) and the Cochrane Library (2011, Issue 12). To identify all relevant RCTs on this subject, the following search terms were used: ('silodosin' OR 'KMD-3213') AND 'lower urinary tract symptoms' AND ('benign prostatic hyperplasia' OR 'benign prostatic hypertrophy'). We also searched the references of the included studies to identify additional potentially relevant studies. The search strategy was not restricted by publication year or language.

The above mentioned search strategy was used to obtain the titles and abstracts of RCTs that were relevant to this review. The titles and abstracts were screened independently by two reviewers, who discarded the studies that were not applicable. The two reviewers independently assessed the titles and abstracts of all identified trials to confirm the fulfilment of the inclusion criteria. Disagreements were resolved in consultation with the third reviewer. The data extraction was performed independently by the same authors using standard data extraction forms. The quality of the included randomised trials was assessed using the Cochrane Collaboration's tool. ${ }^{17}$

\section{Inclusion criteria}

Only those RCTs in which men with BPH were randomized to receive either silodosin or another medical treatment for LUTS were identified. However, randomized crossover studies were excluded.

\section{Outcome measures}

Our outcome measures were the change from baseline for the International Prostate Symptom Score (IPSS), quality of life (QoL) score, peak urine maximum flow rate $\left(\mathrm{Q}_{\max }\right)$ and $\mathrm{QoL}$ related to urinary symptoms and adverse effects.

\section{Intervention types}

The interventions were silodosin versus placebo or other medical treatment.

\section{Statistical analysis}

We analysed the data using Review Manager 5.1 (The Nordic Cochrane Centre, The Cochrane Collaboration, 2011, Copenhagen, Denmark) and then extracted and pooled the data for the summary estimates. According to the Cochrane guideline, ${ }^{17}$ we combined the data on dichotomous outcomes using the Mantel-Haenszel relative risk method (RR). For continuous outcomes, we used the inverse variance mean difference (MD) method and 95\% confidence intervals (CIs). We used the $\chi^{2}$ statistic to assess the heterogeneity between trials and the $I^{2}$ statistic to assess the extent of inconsistency. We used a fixed effect model to calculate the summary estimates and their 95\% CIs unless there was significant heterogeneity. In the case of significant heterogeneity, the results were confirmed using a random effects statistical model. If the data were not depicted by mean \pm s.d., the standard deviation was estimated using the Cochrane statistical method. ${ }^{17}$

\section{RESULTS}

The combined search strategies identified four RCTs, which included a total of 2504 patients (1109 in the silodosin group, 736 in the placebo group and 659 in the tamsulosin group), which met the inclusion criteria (Figure 1). All studies ${ }^{14-16,18}$ were randomized, double-blind, multicentre studies. The study durations were each 12 weeks. Three ${ }^{14-16}$ of the four studies were placebo-controlled studies. Three ${ }^{14,16,18}$ of the four studies compared $8 \mathrm{mg}$ silodosin with $0.2 \mathrm{mg}$ tamsulosin. However, in one study, ${ }^{16}$ the standard deviation could not be estimated by the statistical method. All studies used $8 \mathrm{mg}$ silodosin for LUTS in men with BPH. One RCT ${ }^{16}$ was conducted in Europe, one $\mathrm{RCT}^{15}$ was conducted in the USA and the two remaining $\mathrm{RCTs}^{14,18}$ were conducted in Asia. The characteristics and quality assessment of the four studies are summarized in Tables 1 and 2.

\section{Silodosin versus placebo}

The IPSS. The pooled results are shown in Figure 2. Three studies reported the change from baseline for the IPSS. The pooled results showed that the silodosin group was significantly higher than placebo group in the change from baseline of the IPSS ( $\mathrm{MD}=-2.78,95 \% \mathrm{CI}$ : $-3.42--2.14, P<0.00001)$. Subsequently, subgroup analysis was performed to explore the storage symptoms and voiding symptoms of the IPSS. The pooled results demonstrated that there was still statistical significance for the storage symptoms and voiding symptoms in the silodosin group compared with the placebo group (storage symptoms: $\mathrm{MD}=-0.85,95 \% \mathrm{CI}:-1.11--0.59, P<0.00001$; voiding symptoms: $\mathrm{MD}=-1.81,95 \% \mathrm{CI}:-2.21--1.42, P<0.00001)$.

The QoL score. The pooled results are shown in Figure 2. Two studies reported the change from baseline for the QoL score. Heterogeneity was observed in the pooled analysis $\left(I^{2}=59 \%\right)$. Therefore, we performed the meta-analysis using the random-effect model. The pooled results showed that the silodosin group was significantly higher than placebo group in the change from baseline for the QoL score $(\mathrm{MD}=-0.42,95 \% \mathrm{CI}:-0.71--0.13, P=0.004)$.

The $Q_{\max }$. The pooled result is shown in Figure 3. Three studies reported the change from baseline for the $\mathrm{Q}_{\max }$. The pooled results showed that the silodosin group was significantly higher than placebo group in the change from baseline for $\mathrm{Q}_{\max }(\mathrm{MD}=1.17,95 \% \mathrm{CI}$ : $0.78-$ $1.57, P<0.00001)$.

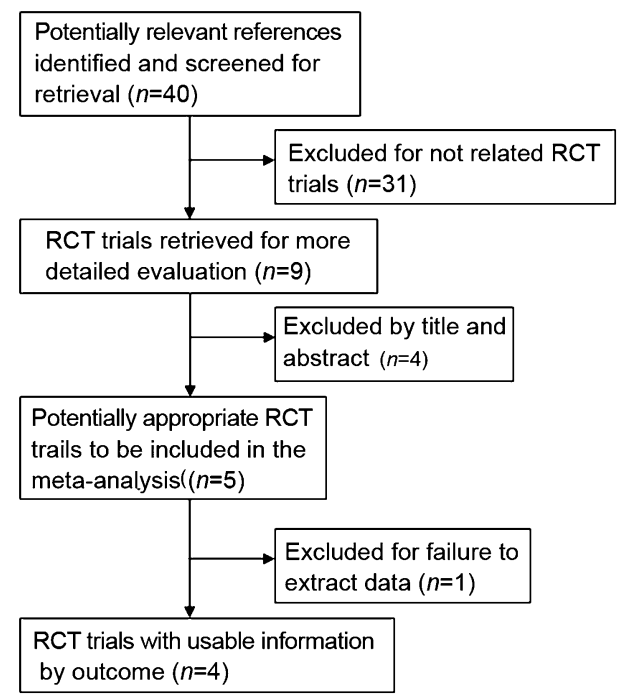

Figure 1 Flow chart of the procedure for selecting randomised controlled trials (RCTs) for analysis. 
Table 1 Baseline characteristics of the included studies

\begin{tabular}{|c|c|c|c|c|c|c|}
\hline Study, year & Intervention (n) & Mean age (year) & Study duration (week) & Total IPSS & QoL score & $Q_{\max }\left(m / \mathrm{s}^{-1}\right)$ \\
\hline \multirow[t]{3}{*}{ Kawabe et al., ${ }^{14} 2006$} & Placebo $(n=89)$ & $65.0 \pm 6.9$ & \multirow[t]{3}{*}{12} & $17.1 \pm 6.1$ & $4.7 \pm 0.8$ & $9.96 \pm 2.65$ \\
\hline & Silodosin $(n=175)$ & $65.4 \pm 7.0$ & & $17.1 \pm 5.7$ & $4.9 \pm 0.8$ & $9.89 \pm 2.72$ \\
\hline & Tamsulosin $(n=192)$ & $65.6 \pm 7.0$ & & $17.0 \pm 5.7$ & $4.7 \pm 0.8$ & $9.43 \pm 2.79$ \\
\hline \multirow[t]{2}{*}{ Marks et al., ${ }^{15} 2009$} & Placebo $(n=457)$ & $64.7 \pm 8.1$ & \multirow[t]{2}{*}{12} & $21.3 \pm 4.9$ & \multirow[t]{2}{*}{ None } & $8.9 \pm 2.8$ \\
\hline & Silodosin $(n=466)$ & $64.6 \pm 8.1$ & & $21.3 \pm 5.1$ & & $8.7 \pm 2.6$ \\
\hline \multirow[t]{3}{*}{ Chapple et al., ${ }^{16} 2011$} & Placebo $(n=190)$ & $66.0 \pm 7.37$ & \multirow[t]{3}{*}{12} & $19.3 \pm 4.33$ & $4.0 \pm 1.00$ & $10.32 \pm 2.816$ \\
\hline & Silodosin $(n=381)$ & $65.8 \pm 7.70$ & & $19.1 \pm 4.23$ & $3.9 \pm 1.01$ & $10.78 \pm 2.726$ \\
\hline & Tamsulosin $(n=384)$ & $65.9 \pm 7.41$ & & $18.9 \pm 4.37$ & $3.9 \pm 1.09$ & $10.27 \pm 2.726$ \\
\hline \multirow[t]{2}{*}{ Yu et al., ${ }^{18} 2011$} & Silodosin $(n=87)$ & $67.5 \pm 9.3$ & \multirow[t]{2}{*}{12} & $19.3 \pm 4.5$ & $3.8 \pm 0.8$ & $10.3 \pm 2.8$ \\
\hline & Tamsulosin $(n=83)$ & $65.0 \pm 8.8$ & & $19.8 \pm 4.5$ & $3.7 \pm 0.8$ & $10.6 \pm 2.8$ \\
\hline
\end{tabular}

Abbreviations: IPSS, International Prostate Symptom Score; QoL, quality of life; $Q_{\max }$, peak urine maximum flow rate.

The QoL score related to urinary symptoms. The pooled results are shown in Figure 4. Two studies reported the QoL related to urinary symptoms. The pooled results showed that the patients felt more satisfaction in the silodosin group than in the placebo group (delighted, pleased or mostly satisfied: $\mathrm{RR}=1.36,95 \% \mathrm{CI}$ : $1.16-1.59$, $P=0.0001$; mixed: $\mathrm{RR}=1.20,95 \% \mathrm{CI}: 1.00-1.44, P=0.05$; mostly dissatisfied, unhappy or terrible: $\mathrm{RR}=0.72,95 \%$ CI: $0.64-0.81$, $P<0.00001)$.

The adverse effects. The pooled results are shown in Figure 5. All included studies indicated that the most common drug-related adverse events were ejaculation disorder, headache, dizziness and diarrhoea. Our pooled results showed that the incidence of ejaculation disorder was significantly higher in the silodosin group than in the placebo group $(\mathrm{RR}=26.11,95 \% \mathrm{CI}: 12.12-56.22, P<0.00001)$, whereas there was no significant difference in the incidences of headache, dizziness and diarrhoea (headache: $\mathrm{RR}=1.54,95 \% \mathrm{CI}$ : 0.83 2.87, $P=0.17$; dizziness: $\mathrm{RR}=2.02$, 95\% CI: $0.97-4.23, P=0.06$; diarrhoea: $\mathrm{RR}=1.57,95 \%$ CI: $0.78-3.16, P=0.20)$.

\section{Silodosin versus tamsulosin}

To explore the efficacy and safety of silodosin, two studies compared $8 \mathrm{mg}$ silodosin with $0.2 \mathrm{mg}$ tamsulosin with respect to the IPSS, QoL score, $\mathrm{Q}_{\max }$ and adverse effects. Our pooled results showed that the silodosin group was superior to the $0.2 \mathrm{mg}$ tamsulosin group with respect to the IPSS and QoL score (IPSS: $\mathrm{MD}=-1.14,95 \% \mathrm{CI}$ : $-2.11--0.18, P=0.02$; QoL score: $\mathrm{MD}=-0.26$, 95\% CI: $-0.47-$ $-0.05, P=0.02$ ) (Figure 6), whereas the silodosin group was inferior to the $0.2 \mathrm{mg}$ tamsulosin group with respect to the $\mathrm{Q}_{\max }(\mathrm{MD}=-0.85$, 95\% CI: $-1.49--0.21, P=0.01$ ) (Figure 7). However, there were no significant differences in the incidence of ejaculation disorder and dizziness $(\mathrm{RR}=3.62,95 \% \mathrm{CI}: 0.22-59.93, P=0.37 ; \mathrm{RR}=1.33,95 \%$ CI: $0.30-6.00, P=0.71$, respectively) (Figure 8). In the IPSS subscores, the change in the voiding symptoms from baseline was significantly different between the silodosin and $0.2 \mathrm{mg}$ tamsulosin groups $(\mathrm{MD}=-0.78,95 \% \mathrm{CI}:-1.48--0.07, P=0.03)$. There was no difference in the change in the storage symptoms from baseline between the silodosin and $0.2 \mathrm{mg}$ tamsulosin groups $(\mathrm{MD}=-0.32,95 \% \mathrm{CI}$ : $-0.75-0.11, P=0.15$ ), but the improvement in the storage symptoms in the silodosin group was better than in the $0.2 \mathrm{mg}$ tamsulosin group (Figure 6).

\section{DISCUSSION}

To our knowledge, this meta-analysis is the first to evaluate the efficacy and safety of silodosin for the treatment of $\mathrm{BPH}$. BPH is the most important cause of LUTS in males, and $50 \%$ of men with BPH complain about LUTS. ${ }^{19}$ Male LUTS can be classified into three categories, including voiding (hesitancy, slow stream, intermittency, incomplete emptying), storage (frequency, urgency, nocturia, urge urinary incontinence) and postmicturition (postvoid dribbling).$^{20}$ Moreover, both the voiding and storage symptoms have a significant impact on the overall QoL. ${ }^{21}$ To date, alpha ${ }_{1}$-adrenergic receptor antagonists are one of the most common treatments for BPH due to LUTS. Silodosin is a new alpha $_{1 \mathrm{~A}}$-blocker and has been approved by the US Food and Drug Administration for the treatment of $\mathrm{BPH}$ since October 2008. ${ }^{22}$ However, the efficacy and safety of silodosin were unclear. Therefore, we combined subject searching with random searching to obtain relevant articles and used meta-analysis to evaluate the efficacy and safety of silodosin for the treatment of LUTS/BPH. In our study, we found that compared with placebo, silodosin can significantly improve the IPSS, QoL score and $\mathrm{Q}_{\max }$. Among the adverse effects, ejaculation disorder was the most common adverse effect $(21.9 \%$ versus $0.8 \%$, respectively). Moreover, compared with $0.2 \mathrm{mg}$ tamsulosin, silodosin can significantly improve the IPSS, QoL score and $\mathrm{Q}_{\max }$. However, the incidence of ejaculation disorder was higher.

In this meta-analysis, the results indicate that silodosin was better than placebo and not inferior to tamsulosin, which was verified by the reduction in total IPSS. The difference in the change in the total IPSS between the silodosin and tamsulosin groups was -1.4 (95\% CI: $-2.11-0.18$, respectively). Therefore, $8 \mathrm{mg}$ silodosin is considered to be at least as effective as $0.2 \mathrm{mg}$ tamsulosin, the recommended dosage regimen in Asian countries. Moreover, with respect to subjective symptoms, silodosin was effective in reducing not only the voiding symptoms but also the storage symptoms. In addition, the change in

Table 2 Methodological quality of the included studies

\begin{tabular}{|c|c|c|c|c|c|c|}
\hline Study & $\begin{array}{c}\text { Adequate sequence } \\
\text { generation? }\end{array}$ & $\begin{array}{c}\text { Allocation } \\
\text { concealment }\end{array}$ & Blinding & Incomplete outcome data? & $\begin{array}{l}\text { Selective outcome } \\
\text { reporting }\end{array}$ & $\begin{array}{c}\text { Other sources of } \\
\text { bias }\end{array}$ \\
\hline Kawabe et al., ${ }^{14} 2006$ & Unclear & Unclear & Double-blind & Yes & Unclear & Unclear \\
\hline Marks et al.., ${ }^{15} 2009$ & Permuted block design & Unclear & Double-blind & Yes & Unclear & Unclear \\
\hline Chapple et al., ${ }^{16} 2011$ & Unclear & Unclear & Double-blind & Yes & Unclear & Unclear \\
\hline Yu et al., ${ }^{18} 2011$ & Unclear & Unclear & Double-blind & Yes & Unclear & Unclear \\
\hline
\end{tabular}




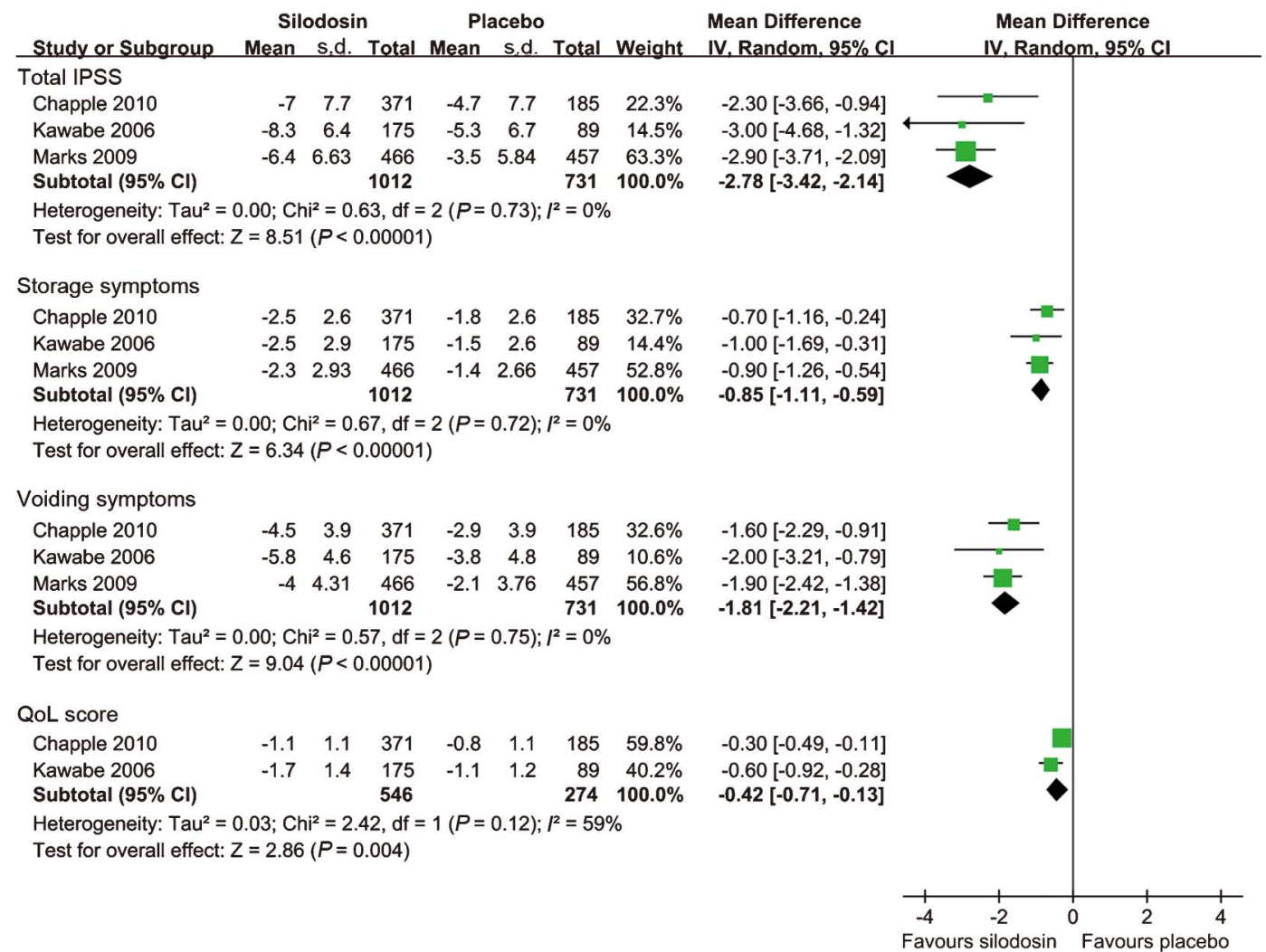

Figure 2 Results of the meta-analysis on the change from baseline for the IPSS and QoL score (silodosin versus placebo). IPSS, International Prostate Symptom Score; QoL, quality of life.

the QoL score from baseline was significantly different between the silodosin group and the placebo or $0.2 \mathrm{mg}$ tamsulosin group.

Alpha $_{1}$-ARs are a family of $\mathrm{G}$ protein-coupled receptors. The binding of norepinephrine and epinephrine induces phospholipase $\mathrm{C}$ activation, leading to the generation of secondary messengers, including inositol triphosphate and diacylglycerol. Finally, this binding induces an increase in the intracellular calcium levels and smooth muscle contraction. ${ }^{23}$ As is well known, prostate contraction is the main cause of LUTS due to BPH and is predominantly mediated by alpha $_{1 \mathrm{~A}}-\mathrm{AR} .^{24,25}$ Therefore, the blockage of alpha ${ }_{1 \mathrm{~A}}$-AR induces prostatic and urethral smooth muscle relaxation and may improve the voiding symptoms. Yokoyama et al. ${ }^{26}$ demonstrated that the selective alpha $\mathrm{aA}_{1 \mathrm{~A}}$-blocker silodosin exerts inhibitory effects on detrusor overactivity by modulating C-fibre afferent activity using animal models. Consequently, alpha $\mathrm{1A}_{\mathrm{A}}$-blockade can improve the storage symptoms.
Wilt and colleagues ${ }^{9}$ assessed the efficacy and safety of tamsulosin for the treatment of BPH. In the tamsulosin studies, 14 studies involving 4122 subjects were included; in these studies, the placebo-controlled study duration lasted less than 13 weeks, and the mean age of the subjects was 64 years. The pooled results showed that the weighted mean differences for the mean change from baseline for the Boyarsky symptom score for the $0.4 \mathrm{mg}$ and $0.8 \mathrm{mg}$ doses of tamsulosin relative to placebo were -1.1 and -1.6 points, respectively. The weighted mean differences for the mean change from baseline in peak urine flow were 1.1 and $1.1 \mathrm{ml} \mathrm{s}^{-1}$ for 0.4 and $0.8 \mathrm{mg}$, respectively. In the included studies, our pooled results showed that the MD for the mean change from baseline for IPSS for the $8 \mathrm{mg}$ dose of silodosin relative to placebo was -2.78 points. The MD for the mean change from baseline in the peak urine flow was $1.17 \mathrm{ml} \mathrm{s}^{-1}$ for $8 \mathrm{mg}$ silodosin. Moreover, we also assessed the efficacy and safety of silodosin for the treatment of BPH compared with tamsulosin. The pooled results indicated that the MD

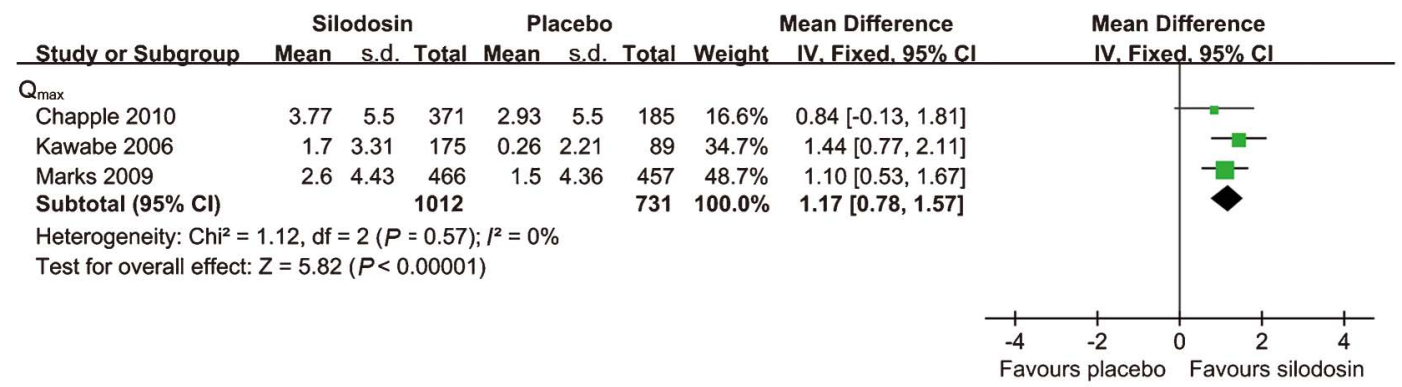

Figure 3 Results of the meta-analysis on the change from baseline for $Q_{\max }$ (silodosin versus placebo). $Q_{\max }$, peak urine maximum flow rate. 


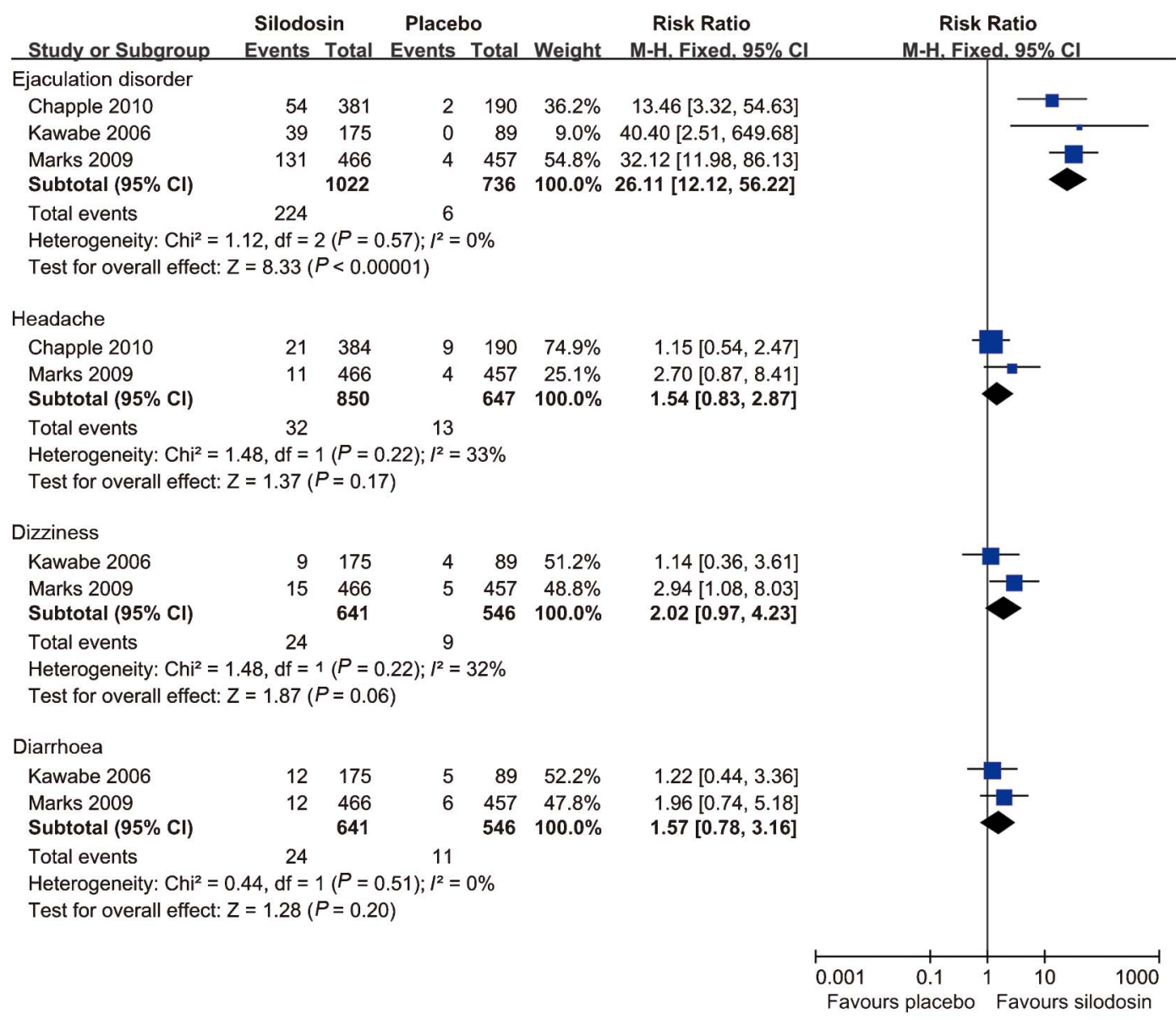

Figure 4 Results of the meta-analysis on the quality of life related to urinary symptoms (silodosin versus placebo).

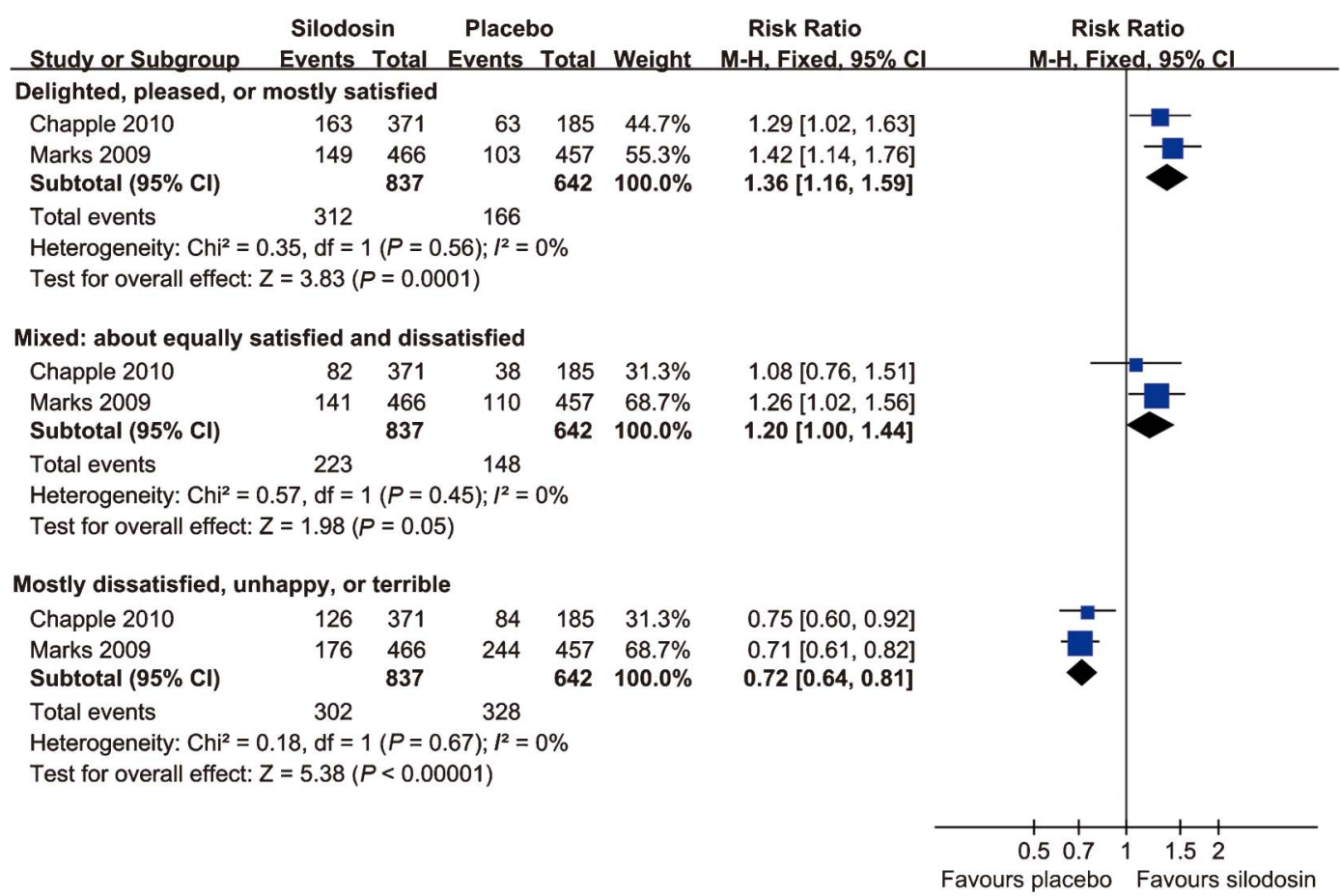

Figure 5 Results of the meta-analysis on the adverse effects (silodosin versus placebo). 


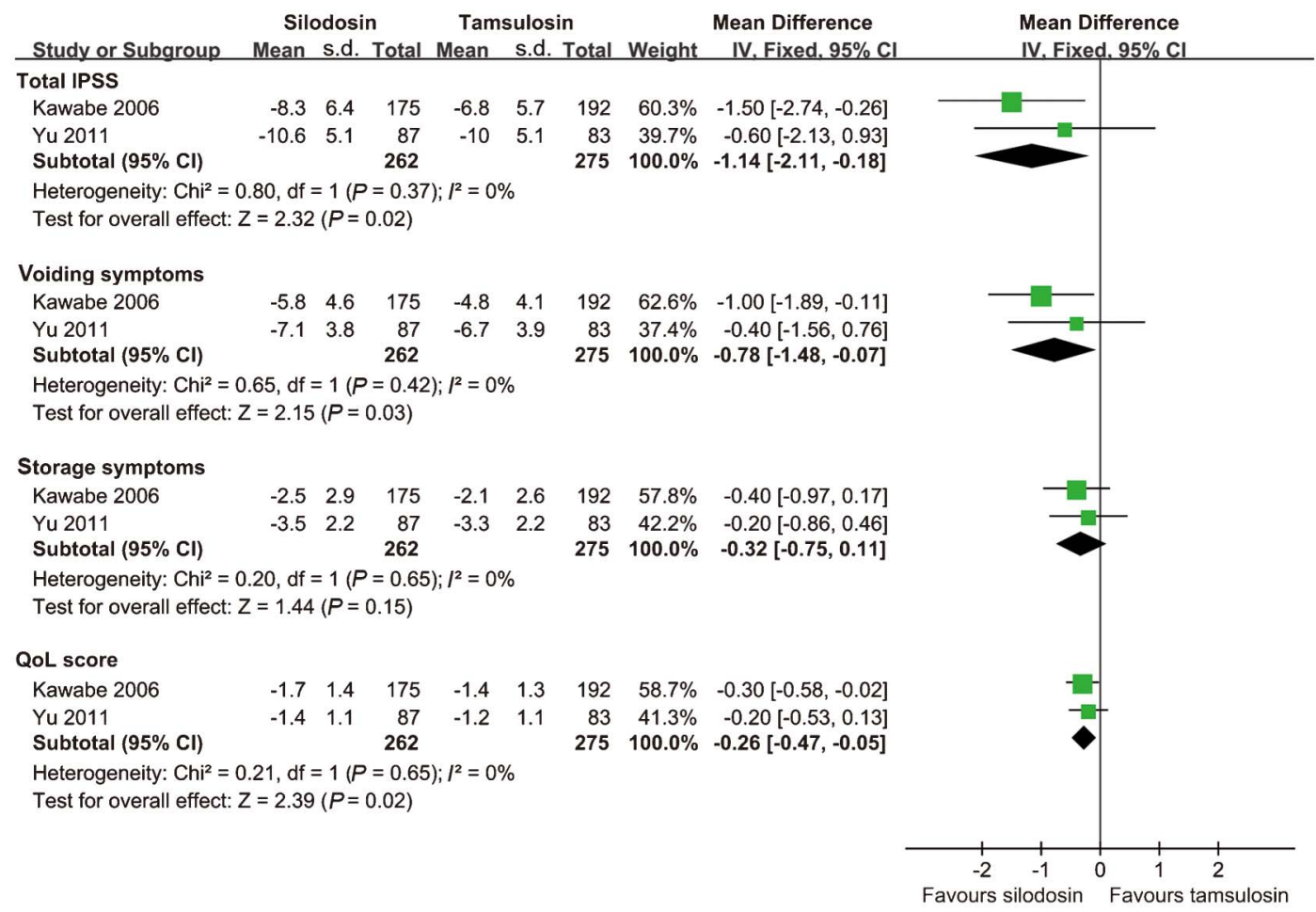

Figure 6 Results of the meta-analysis on the change from baseline for the IPSS and QoL score (silodosin versus tamsulosin). IPSS, International Prostate Symptom Score; QoL, quality of life.

for the mean change from baseline in the IPSS, peak urine flow and QoL score were -1.14 points, $-0.85 \mathrm{ml} \mathrm{s}^{-1}$ and -0.26 points, respectively.

In these studies, ${ }^{14,15,18}$ the most commonly reported adverse reaction for silodosin was ejaculation disorder. Compared with $0.2 \mathrm{mg}$ tamsulosin, the incidence of ejaculation disorder was higher (18\% vs. $4 \%$ ) in this meta-analysis. Similarly, Chapple et al. ${ }^{16}$ reported that the frequency of 'ejaculation disorder' was $14.2 \%$ in the silodosin treatment group, which was also significantly higher compared with $2.1 \%$ in the $0.4 \mathrm{mg}$ tamsulosin treatment group. However, Song et al. ${ }^{27}$ reported that the overall incidence of ejaculatory dysfunction was $13.4 \%$ after 12 weeks of $0.2 \mathrm{mg}$ tamsulosin treatment. Retrograde ejaculation is caused by smooth muscle relaxation in the prostate, urethra, bladder neck and vas deferens. The alpha ${ }_{1 \mathrm{~A}}-\mathrm{AR}$ is mainly expressed in the bladder neck, vas deferens and seminal vesicles. ${ }^{23}$ Moreover, Moriyama et al. ${ }^{28}$ showed that the alpha ${ }_{1 \mathrm{~A}}$-AR subtype mediates human vas deferens contraction. Therefore, this adverse effect is explained by the high alpha ${ }_{1 \mathrm{~A}}$-AR subtype selectivity of silodosin. Moreover, Homma et al. ${ }^{29}$ performed a post hoc analysis of the data from a randomized, double-blind, placebo-controlled clinical trial of silodosin in Japan and found that ejaculation disorder caused by selective alpha $\mathrm{IA}_{\mathrm{A}}$-blockers was associated with very large improvements in the lower urinary tract symptoms. The silodosin subgroup with ejaculation failure experienced a greater reduction in the total
IPSS than the silodosin subgroup without ejaculation impairment and the placebo subgroup $(-11.8 v s .-7.2 v s$. -5.3 , respectively). However, there was no difference in the discontinuation rates between the silodosin subgroup with ejaculation disorder and the silodosin subgroup without ejaculation disorder. Thus, ejaculation disorder may be a predictor of the efficacy of the alpha ${ }_{1}$-AR blockade.

Akiyama et al. ${ }^{30}$ demonstrated that silodosin did not induce any negative cardiovascular effects in patients with $\mathrm{BPH}$ in male decerebrate dogs. Tatemichi et al. ${ }^{31}$ indicated that silodosin did not affect blood pressure, heart rate or electrocardiogram in conscious dogs with voiding dysfunction. A recent $\mathrm{RCT}^{16}$ suggested that compared with tamsulosin or placebo, silodosin showed no significant differences in the supine systolic blood pressure, diastolic blood pressure and heart rate. Alpha ${ }_{1 \mathrm{~B}}$-ARs have been demonstrated to mediate both blood vessel contraction and baroreceptor-induced inotropic effects. ${ }^{32-34}$ Moreover, tamsulosin has a higher selectivity for the alpha $\mathrm{ab}_{1 \mathrm{~B}}-\mathrm{AR}$ than silodosin, and thus, the decrease in blood pressure induced by tamsulosin may be mediated by its blocking action on the alpha $\mathrm{a}_{1 \mathrm{~B}}-\mathrm{AR}$ participating in blood vessel contraction and baroreceptor-induced inotropic effects. ${ }^{13,24}$ Therefore, the lack of cardiovascular side effects may be a major advantage of silodosin.

Our meta-analysis had several limitations. First, all included studies are of moderate quality. ${ }^{35}$ Second, the durations of all the included

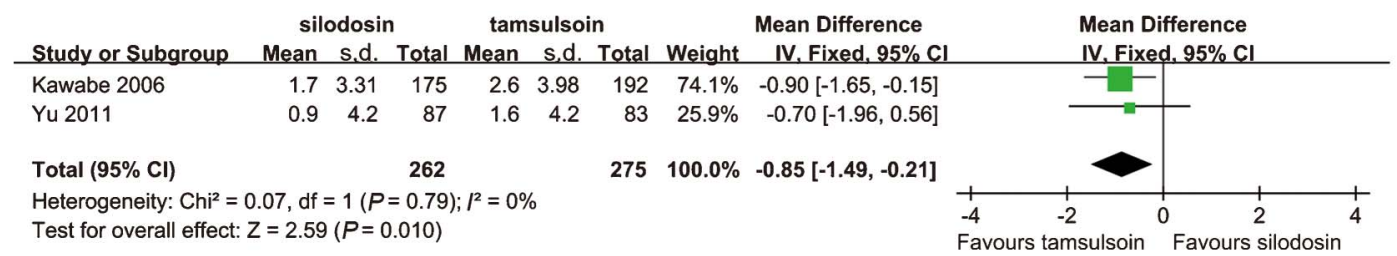

Figure 7 Results of the meta-analysis on the change from baseline for $Q_{\max }$ (silodosin versus tamsulosin). $Q_{\max }$, peak urine maximum flow rate. 


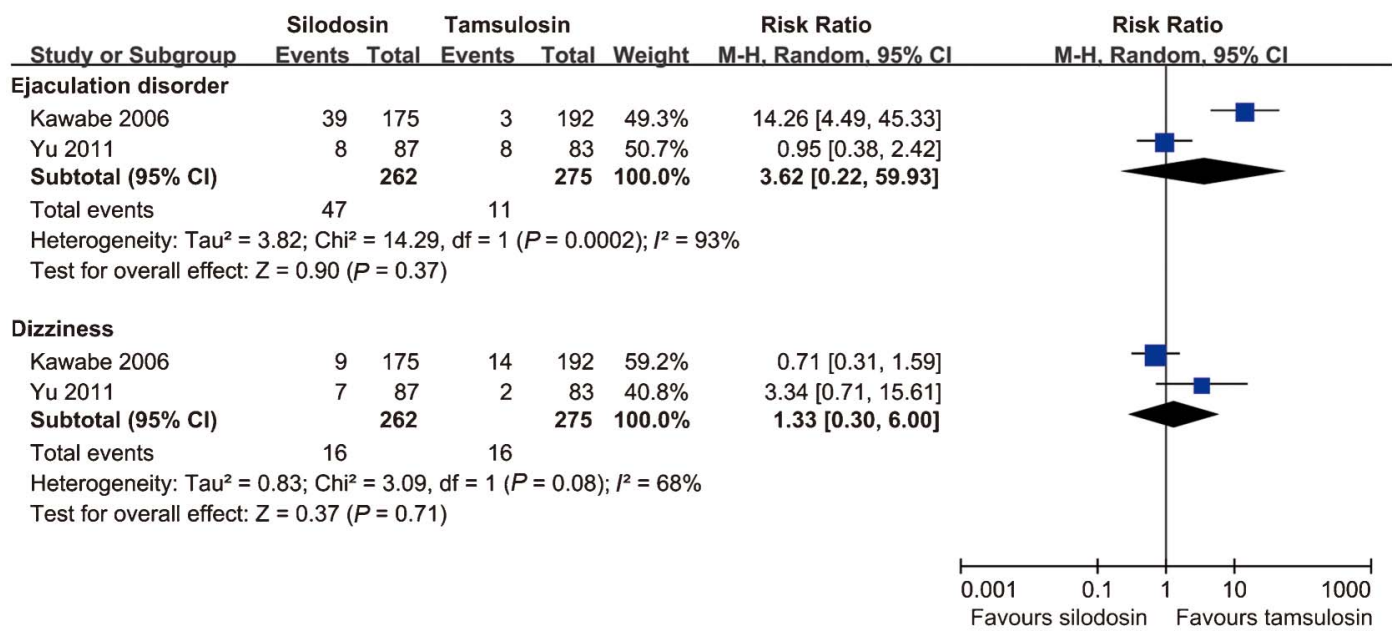

Figure 8 Results of the meta-analysis on the adverse effects (silodosin versus tamsulosin).

studies were short (only 12 weeks). Third, in this meta-analysis, the dose of tamsulosin was $0.2 \mathrm{mg}$ and the dosage regimen of silodosin was $4 \mathrm{mg}$ twice a day, which is commonly used in Asia, but the used dose of tamsulosin is $0.4 \mathrm{mg}$ and the dosage regimen of silodosin is $8 \mathrm{mg}$ once daily in Europe and the United States. These limitations might not allow for a reliable conclusion. Therefore, the findings of this review require a more efficient performance of higher-quality, long-term randomized controlled trials to verify and explore the efficacy and safety of silodosin. In particular, studies that compare $8 \mathrm{mg}$ silodosin with $0.4 \mathrm{mg}$ tamsulosin are needed in the future.

\section{CONCLUSION}

The current meta-analysis suggested that silodosin is an effective therapy for LUTS in men with BPH, but that the incidence of retrograde ejaculate was higher than in the placebo or tamsulosin treatment. In the future, higher-quality and long-term RCTs are needed to verify the findings of this review, and studies that compare $8 \mathrm{mg}$ silodosin with $0.4 \mathrm{mg}$ tamsulosin are also needed.

\section{AUTHOR CONTRIBUTIONS}

$\mathrm{HD}, \mathrm{WD}, \mathrm{ZZH}$ and $\mathrm{ZPW}$ conceived the study, participated in its design, and coordinated and drafted the manuscript. HD, WD, ZZH, HZW and ZPW collected the data. HD, WD and HZW performed the statistical analysis. $\mathrm{HD}, \mathrm{WD}, \mathrm{ZZH}$ and $\mathrm{ZPW}$ participated in the critical revision of the manuscript. All authors read and approved the final manuscript.

\section{COMPETING FINANCIAL INTERESTS}

The authors have no financial or commercial interests related to this study.

\section{ACKNOWLEDGMENTS}

This paper was supported by the Scholarship Award for Excellent Doctoral Student granted by Lanzhou University, China

1 Chapple C. Medical treatment for benign prostatic hyperplasia. BMJ 1992; 304 1198-9.

2 McNicholas T,Kirby R. Benign prostatic hyperplasia and male lower urinary tract symptoms (LUTS). Clin Evid (Online) 2011; pii: 1801.
3 Berry SJ, Coffey DS, Walsh PC, Ewing LL. The development of human benign prostatic hyperplasia with age. J Urol 1984; 132: 474-9.

4 Trueman P, Hood SC, Nayak US, Mrazek MF. Prevalence of lower urinary tract symptoms and self-reported diagnosed 'benign prostatic hyperplasia', and their effect on quality of life in a community-based survey of men in the UK. BJU Int 1999; 83: 410-5.

5 Oelke M, Bachmann A, Descazeaud A, Emberton M, Gravas S et al. Guidelines on the Treatment of Non-neurogenic Male LUTS. Arnhem: EAU; 2011. p8-12.

6 MacDonald R, Wilt TJ. Alfuzosin for treatment of lower urinary tract symptoms compatible with benign prostatic hyperplasia: a systematic review of efficacy and adverse effects. Urology 2005; 66: 780-8.

7 Jonler M, Riehmann M, Bruskewitz RC. Benign prostatic hyperplasia. Current pharmacological treatment. Drugs 1994; 47: 66-81.

8 MacDonald R, Wilt TJ, Howe RW. Doxazosin for treating lower urinary tract symptoms compatible with benign prostatic obstruction: a systematic review of efficacy and adverse effects. BJU Int 2004; 94: 1263-70.

9 Wilt TJ, MacDonald R, Rutks I. Tamsulosin for benign prostatic hyperplasia. Cochrane Database Syst Rev 2003; (1): CD002081.

10 Wilt TJ, Howe RW, Rutks IR, MacDonald R. Terazosin for benign prostatic hyperplasia. Cochrane Database Syst Rev 2002; (4): CD003851.

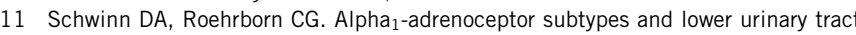
symptoms. Int J Urol 2008; 15: 193-9.

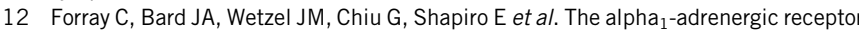
that mediates smooth muscle contraction in human prostate has the pharmacological properties of the cloned human alpha 1c subtype. Mol Pharmacol 1994; 45: 703-8.

13 Tatemichi S, Kobayashi K, Maezawa A, Kobayashi M, Yamazaki Y et al. [Alpha adrenoceptor subtype selectivity and organ specificity of silodosin (KMD-3213).] Yakugaku Zasshi 2006; 126 Spec no.: 209-16.

14 Kawabe K, Yoshida M, Homma Y. Silodosin, a new alpha ${ }_{1 \mathrm{~A}}$-adrenoceptor-selective antagonist for treating benign prostatic hyperplasia: results of a phase III randomized, placebo-controlled, double-blind study in Japanese men. BJU Int 2006; 98: 1019-24.

15 Marks LS, Gittelman MC, Hill LA, Volinn W, Hoel G. Rapid efficacy of the highly selective alpha $\mathrm{A}_{\mathrm{A}}$-adrenoceptor antagonist silodosin in men with signs and symptoms of benign prostatic hyperplasia: pooled results of 2 phase 3 studies. J Urol 2009; 181: 2634-40.

16 Chapple CR, Montorsi F, Tammela TL, Wirth M, Koldewijn E et al. Silodosin therapy for lower urinary tract symptoms in men with suspected benign prostatic hyperplasia: results of an international, randomized, double-blind, placebo- and activecontrolled clinical trial performed in Europe. Eur Urol 2011; 59: 342-52.

17 Higgins. J, Green. S. Cochrane Handbook for Systematic Reviews of Interventions, Version 5.0.2, Updated September 2009. Oxford: The Cochrane Collaboration; 2009.

$18 \mathrm{Yu} \mathrm{HJ}$, Lin AT, Yang SS, Tsui KH, Wu HC et al. Non-inferiority of silodosin to tamsulosin in treating patients with lower urinary tract symptoms (LUTS) associated with benign prostatic hyperplasia (BPH). BJU Int 2011; 108: 1843-8.

19 Wasserman NF. Benign prostatic hyperplasia: a review and ultrasound classification. Radiol Clin North Am 2006; 44: 689-710, viii.

20 Chapple CR, Roehrborn CG. A shifted paradigm for the further understanding, evaluation, and treatment of lower urinary tract symptoms in men: focus on the bladder. Eur Urol 2006; 49: 651-8.

21 Sountoulides P, van Dijk MM, Wijkstra H, de la Rosette JJ, Michel MC. Role of voiding and storage symptoms for the quality of life before and after treatment in men with voiding dysfunction. World J Urol 2010; 28: 3-8.

22 Schilit S, Benzeroual KE. Silodosin: a selective alpha ${ }_{1 A}$-adrenergic receptor antagonist for the treatment of benign prostatic hyperplasia. Clin Ther 2009; 31: 2489-502. 
23 Rossi M, Roumeguere T. Silodosin in the treatment of benign prostatic hyperplasia. Drug Des Devel Ther 2010; 4: 291-7.

24 Michel MC, Vrydag W. Alpha $1^{-}$, alpha $2^{-}$and beta-adrenoceptors in the urinary bladder, urethra and prostate. Br J Pharmacol 2006; 147(Suppl 2)S88-119.

25 Roehrborn CG. Efficacy of alpha-adrenergic receptor blockers in the treatment of male lower urinary tract symptoms. Rev Urol 2009; 11: S1-8.

26 Yokoyama O, Ito H, Aoki Y, Oyama N, Miwa Y et al. Selective alpha1A-blocker improves bladder storage function in rats via suppression of $\mathrm{C}$-fiber afferent activity. World $\mathrm{J}$ Urol 2010; 28: 609-14.

27 Song SH, Son H, Kim KT, Kim SW, Moon du G et al. Effect of tamsulosin on ejaculatory function in BPH/LUTS. Asian J Androl 2011; 13: 846-50.

28 Moriyama N, Nasu K, Takeuchi T, Akiyama K, Murata S et al. Quantification and distribution of alpha 1-adrenoceptor subtype mRNAs in human vas deferens: comparison with those of epididymal and pelvic portions. Br J Pharmacol 1997; 122: $1009-14$

29 Homma Y, Kawabe K, Takeda M, Yoshida M. Ejaculation disorder is associated with increased efficacy of silodosin for benign prostatic hyperplasia. Urology 2010; 76: 1446-50.
30 Akiyama K, Noto H, Nishizawa O, Sugaya K, Yamagishi R et al. Effect of KMD-3213, an alpha ${ }_{1 \mathrm{~A}}$-adrenoceptor antagonist, on the prostatic urethral pressure and blood pressure in male decerebrate dogs. Int J Urol 2001; 8: 177-83.

31 Tatemichi S, Kiguchi S, Kobayashi M, Yamazaki Y, Shibata N et al. Cardiovascular effects of the selective alphal ${ }_{A}$-adrenoceptor antagonist silodosin (KMD-3213), a drug for the treatment of voiding dysfunction. Arzneimittelforschung 2006; 56: 682-7.

32 Hatano A, Takahashi H, Tamaki M, Komeyama T, Koizumi T et al. Pharmacological evidence of distinct alpha 1-adrenoceptor subtypes mediating the contraction of human prostatic urethra and peripheral artery. Br J Pharmacol 1994; 113: 723

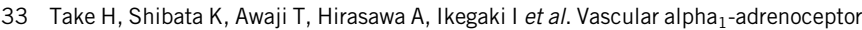

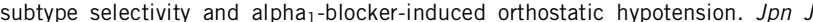
Pharmacol 1998; 77: 61-70.

34 Townsend SA, Jung AS, Hoe YS, Lefkowitz RY, Khan SA et al. Critical role for the alpha-1B adrenergic receptor at the sympathetic neuroeffector junction. Hypertension 2004; 44: 776-82.

35 Balshem H, Helfand M, Schunemann HJ, Oxman AD, Kunz R et al. GRADE guidelines: 3. Rating the quality of evidence. J Clin Epidemiol 2011; 64: 401-6. 